\title{
Precipitate coarsening in multicomponent systems
}

\author{
P.E.J. Rivera-Díaz-del-Castillo * \\ Department of Materials Science and Metallurgy, University of Cambridge, Pembroke Street, Cambridge CB2 3QZ, UK \\ Received 10 March 2001; accepted 12 March 2002
}

\begin{abstract}
Expressions to obtain composition shifts due to capillarity are presented when the chemical potential shifts of the precipitate phase are taken into account. These are applied to calculate the precipitate coarsening kinetics in multicomponent systems, showing dependence on the solution thermodynamics followed by the continuous phase. (C) 2002 Published by Elsevier Science Ltd. on behalf of Acta Materialia Inc.
\end{abstract}

Keywords: Kinetics; Phase transformations; Coarsening

\section{Introduction}

The theory of precipitate coarsening developed by Lifshitz and Slyozov (LS) [1] and Wagner [2] has recently been extended by Umanstev and Olson [3] to incorporate multicomponent effects; they followed a method consistent with LS and Marqusee and Ross (MR) [4], which used a time scaling technique to derive the power law time dependence and distribution function for the size of the particles of the new phase. In an elegant treatment, Morral and Purdy [5] have extended the results form Umanstev and Olson to include off diagonal terms in the diffusivity.

In theories of precipitate growth and coarsening it is commonly assumed that there is a negligible change in the chemical potential and composition

\footnotetext{
${ }^{*}$ Present address: Department of Materials Science and Technology, Delft Univeristy of Technology, Rotterdamseweg 137, 2628 AL Delft, The Netherlands.

E-mail addresses: p.rivera@tnw.tudelft.nl,pejr2@cam.ac.uk (P.E.J. Rivera-Díaz-del-Castillo).
}

of the precipitate phase. Trivedi [6] has pointed out that this leads to a violation of the equilibrium conditions when a curvature is present; Kulkarni and DeHoff [7] have recognised this, and provided the expressions that account for chemical potential shifts of the precipitate phase in a unary system.

The aim of the present work is to extend the Gibbs-Thomson effect to multicomponent systems allowing changes in chemical potential and composition shifts of the precipitate phase, and to apply this to the theory of precipitate coarsening assessing the effects that this produces.

\section{Gibbs-Thomson effect in multicomponent systems}

Fig. 1 shows the changes in chemical potential and composition in a matrix $(\alpha)$ and a precipitate $(\beta)$ phase for a binary non-dilute system. When the particles are characterised by a curvature $1 / R$, equilibrium demands the simultaneous satisfaction of

$\mu_{1}^{\alpha}(R)=\mu_{1}^{\beta}(R) \quad$ and $\quad \mu_{2}^{\alpha}(R)=\mu_{2}^{\beta}(R)$ 


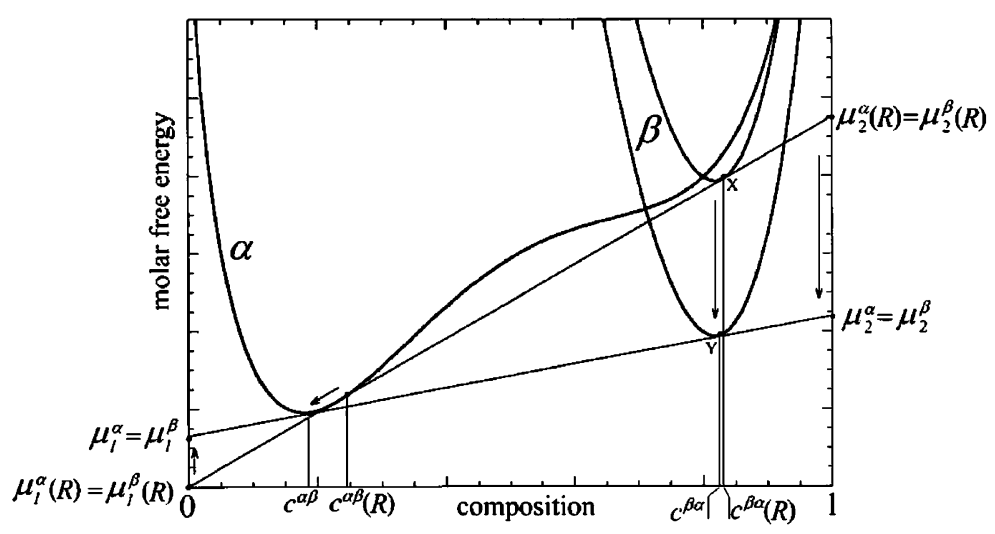

Fig. 1. To illustrate chemical potential and composition shifts during coarsening [3].

which can be expressed as [8]

$$
\begin{aligned}
& \left(\frac{\partial \mu_{1}^{\alpha}}{\partial c_{1}}\right)_{T, P, c_{2}} \Delta c_{1}^{\alpha}=\left(\frac{\partial \mu_{1}^{\beta}}{\partial c_{1}}\right)_{T, P, c_{2}} \Delta c_{1}^{\beta}+\frac{2 \gamma \bar{V}_{1}^{\beta}}{R} \\
& \left(\frac{\partial \mu_{2}^{\alpha}}{\partial c_{2}}\right)_{T, P, c_{1}} \Delta c_{2}^{\alpha}=\left(\frac{\partial \mu_{2}^{\beta}}{\partial c_{2}}\right)_{T, P, c_{1}} \Delta c_{2}^{\beta}+\frac{2 \gamma \bar{V}_{2}^{\beta}}{R}
\end{aligned}
$$

where the derivatives are obtained for constant temperature $(T)$, pressure $(P)$ and the indicated concentrations; $\gamma, \bar{V}_{1}^{\beta}$ and $\bar{V}_{2}^{\beta}$ are the interface energy per unit area, and the $\beta$ phase partial molar volumes of components 1 and 2, respectively. $\Delta c_{i}^{\alpha}=c_{i}^{\alpha \beta}(R)-c_{i}^{\alpha \beta}$ and $\Delta c_{i}^{\beta}=c_{i}^{\beta \alpha}(R)-c_{i}^{\beta \alpha}$ measure the differences between the composition when a curvature is present in $\alpha$ and $\beta$ phases $\left(c_{i}^{\alpha \beta}(R)\right.$ and $c_{i}^{\beta \alpha}(R)$, respectively) and in its absence $\left(c_{i}^{\alpha \beta}, c_{i}^{\beta \alpha}\right)$ for $i=1,2$.

The terms $\left(\partial \mu_{1}^{\beta} / \partial c_{1}\right)_{T, P, c_{2}} \Delta c_{1}^{\beta}$ and $\left(\partial \mu_{2}^{\beta} / \partial c_{2}\right)_{T, P, c_{1}}-$ $\Delta c_{2}^{\beta}$ have been neglected in previous theories of precipitate coarsening, which implies no change in chemical potential of the $\beta$ phase due to a curvature change [6,7]. Furthermore, it is known that in the coarsening process of alloys such as steels [911], there is a composition change of the precipitate phase, which causes a shift in the chemical potential of the $\beta$ phase.

Matrix algebra can be used to express Eqs. (2) and (3). In the notation used here a matrix $\mathbf{A}$ can be expressed as $(\mathbf{A}],[\mathbf{A}]$ or $[\mathbf{A})$, which refer to a row, square or column matrix. Thus the GibbsThomson effect can be written as

$$
\left(c^{\beta \alpha}\right]\left[\mu^{\alpha}\right]\left[\Delta c^{\alpha}\right)=\left(c^{\beta \alpha}\right]\left[\mu^{\beta}\right]\left[\Delta c^{\beta}\right)+\frac{2 \gamma V_{\mathrm{m}}^{\beta}}{R}
$$

where $V_{\mathrm{m}}^{\beta}$ is the molar volume of the $\beta$ phase and

$$
\left[\mu^{\alpha}\right]=\left(\frac{\partial \mu_{i}^{\alpha}}{\partial c_{j}}\right)_{T, P, c_{k}}, \quad\left[\mu^{\beta}\right]=\left(\frac{\partial \mu_{i}^{\beta}}{\partial c_{j}}\right)_{T, P, c_{k}} .
$$

The extension to a system of $i, j=1,2, \ldots, n$ components is immediate.

Eq. (4) and the forthcoming equations can be reduced to $n-1$ independent variables by setting

$\sum_{i=1}^{n} c_{i}=1$

\section{Coarsening rate}

Following Morral and Purdy [5], the mass conservation condition at the interface of a particle of radius $R$ yields

$\left[\Delta c^{\alpha \beta}\right) \frac{\mathrm{d} R}{\mathrm{~d} t}+\frac{[D]\left[\Delta \bar{c}^{\alpha}\right)}{R}=0$

where $\left[\Delta c^{\alpha \beta}\right)=\left[c^{\beta \alpha}(R)-c^{\alpha \beta}(R)\right),\left[\Delta \bar{c}^{\alpha}\right)=\left[c^{\alpha \beta}(R)-\right.$ $\left.\bar{c}^{\alpha}\right)$ where $\left[\bar{c}^{\alpha}\right)$ is the matrix far field concentration and $[D]=D_{i j}$ is the square diffusivity matrix. Eq. (6) can be multiplied by $\left(c^{\beta \alpha}\right]\left[\mu^{\alpha}\right][D]^{-1}$ form which the term $\left(c^{\beta \alpha}\right]\left[\mu^{\alpha}\right]\left[\Delta \bar{c}^{\alpha}\right)$ can be obtained from the Gibbs-Thomson Eq. (4) when this is expressed as 


$$
\begin{gathered}
\left(c^{\beta \alpha}\right]\left[\mu^{\alpha}\right]\left[\Delta \bar{c}^{\alpha}\right)+\left(c^{\beta \alpha}\right]\left[\mu^{\alpha}\right]\left[\Delta \bar{c}^{\alpha \beta}\right) \\
=\left(c^{\beta \alpha}\right]\left[\mu^{\beta}\right]\left[\Delta c^{\beta}\right)+\frac{2 \gamma V_{\mathrm{m}}^{\beta}}{R}
\end{gathered}
$$

where $\left[\Delta \bar{c}^{\alpha \beta}\right)=\left[\bar{c}^{\alpha}-c^{\alpha \beta}\right)$, this can be substituted in Eq. (6) to give

$$
\begin{aligned}
\left(c^{\beta \alpha}\right]\left[\mu^{\alpha}\right][D]^{-1}\left[\Delta c^{\alpha \beta}\right) \frac{\mathrm{d} R}{\mathrm{~d} t}= & \frac{1}{R}\left(\left(c^{\beta \alpha}\right]\left[\mu^{\alpha}\right]\left[\Delta \bar{c}^{\alpha \beta}\right)\right. \\
& \left.-\left(c^{\beta \alpha}\right]\left[\mu^{\beta}\right]\left[\Delta c^{\beta}\right)-\frac{2 \gamma V_{\mathrm{m}}^{\beta}}{R}\right) .
\end{aligned}
$$

It is seen that the critical radius at which a particle dissolves is given by

$R_{\mathrm{c}}=\frac{2 \gamma V_{\mathrm{m}}^{\beta}}{\left(c^{\beta \alpha}\right]\left[\mu^{\alpha}\right]\left[\Delta \bar{c}^{\alpha \beta}\right)-\left(c^{\beta \alpha}\right]\left[\mu^{\beta}\right]\left[\Delta c^{\beta}\right)}$

which includes the term $\left(c^{\beta \alpha}\right]\left[\mu^{\beta}\right]\left[\Delta c^{\beta}\right)$ in the denominator which is lacking in Umanstev and Olson's expression for critical radius [3], this accounts for the $\beta$ phase energy contribution to keep the particle in equilibrium with the matrix as a curvature is present.

To solve Eq. (8) it is recognised that $\Delta(t)=$ $\left(c^{\beta \alpha}\right]\left[\mu^{\alpha}\right]\left[\Delta \bar{c}^{\alpha \beta}\right)$ is a function of time $[3,5]$ and $\left(c^{\beta \alpha}\right]\left[\mu^{\beta}\right]\left[\Delta c^{\beta}\right)$ depends on $R$ only; the functional dependence of the last term can be approximated as

$$
\left(c^{\beta \alpha}\right]\left[\mu^{\beta}\right]\left[\Delta c^{\beta}\right)=\left(c^{\beta \alpha}\right]\left[\mu^{\beta}\right]\left[\Delta c_{\mathrm{r}}^{\beta}\right) \frac{\bar{R}_{\mathrm{r}}}{R}
$$

where $\bar{R}_{\mathrm{r}}$ is an average reference particle radius and $\left[\Delta c_{\mathrm{r}}^{\beta}\right)$ the difference in concentration between a $\beta$ particle of this radius and that of an infinite radius or $\left[\Delta c_{\mathrm{r}}^{\beta}\right)=\left[c^{\beta \alpha}\left(\bar{R}_{\mathrm{r}}\right)-c^{\beta \alpha}\right)$. Eq. (10) describes the locus of the points that join $X$ and $Y$ in Fig. 1, and on substitution in Eq. (8) gives

$\boldsymbol{\Phi} \frac{\mathrm{d} R}{\mathrm{~d} t}=\frac{1}{R}\left(\Delta(t)-\frac{\Omega}{R}\right)$

where $\boldsymbol{\Phi}=\left(c^{\beta \alpha}\right]\left[\mu^{\alpha}\right][D]^{-1}\left[\Delta c^{\alpha \beta}\right)$ characterises the coarsening resistance of the material and $\Omega=$ $2 \gamma V_{\mathrm{m}}^{\beta}+\left(c^{\beta \alpha}\right]\left[\mu^{\beta}\right]\left[\Delta c_{\mathrm{r}}^{\beta}\right) \bar{R}_{\mathrm{r}}$ the total energy increase of $\beta$ phase as a curvature is present.

The second element of the theory is mass balance of the alloy, this can be expressed as $c_{j}^{0}=(1-\varphi) \bar{c}_{j}^{\alpha}+\varphi c_{j}^{\beta \alpha}(R)$,

$\varphi=\frac{4 \pi}{3 V_{\mathrm{m}}^{\beta}} \int_{0}^{\infty} R^{3} f(R, t) \mathrm{d} R$

for $j=1,2, \ldots, n$ components, where $\varphi$ is the volume fraction of $\beta$ phase and $f(R, t)$ the particle radius distribution function. Subtracting $c_{i}^{\alpha \beta}$ from Eq. (12) and multiplying it by $c^{\beta \alpha} \mu_{i j}^{\alpha}$ it is obtained

$$
\begin{aligned}
c_{i}^{\beta \alpha} \mu_{i j}^{\alpha}\left(c_{j}^{0}-c_{j}^{\alpha \beta}\right)= & (1-\varphi) c_{i}^{\beta \alpha} \mu_{i j}^{\alpha}\left(c_{j}^{\alpha}-c_{j}^{\alpha \beta}\right) \\
& +\varphi c_{i}^{\beta \alpha} \mu_{i j}^{\alpha}\left(c_{j}^{\beta \alpha}-c_{j}^{\alpha \beta}\right) \\
& +\varphi c_{i}^{\beta \alpha} \mu_{i j}^{\alpha}\left(c_{j}^{\beta \alpha}(R)-c_{j}^{\beta \alpha}\right)
\end{aligned}
$$

or

$\Delta_{0}=(1-\varphi) \Delta(t)+\varphi \Gamma+\varphi \Lambda$

where $\Delta_{0}=\left(c^{\beta \alpha}\right]\left[\mu^{\alpha}\right]\left[\bar{c}_{0}^{\alpha \beta}\right)$ is the initial cumulative supersaturation of the alloy before precipitation starts, $\Gamma=\left(c^{\beta \alpha}\right]\left[\mu^{\alpha}\right]\left[\Delta c^{\alpha \beta}\right)$ is a time-independent scalar that accounts for the interactions of the system at equilibrium $[3,12], \quad \Lambda=\left(c^{\beta \alpha}\right]\left[\mu^{\alpha}\right]\left[\Delta c^{\beta}\right)$ characterises the binary interactions of the solute concentration increment in $\beta$ phase as curvature changes, this is a function of curvature and can be approximated for a reference radius and composition shift as

$\Delta_{0}=(1-\varphi) \Delta(t)+\varphi \Gamma+\varphi \Lambda_{\mathrm{r}} \frac{\bar{R}_{\mathrm{r}}}{R}$

where $\Lambda_{\mathrm{r}}=\left(c^{\beta \alpha}\right]\left[\mu^{\alpha}\right]\left[\Delta c_{\mathrm{r}}^{\beta}\right)$.

After long time coarsening, excess solute will vanish in the matrix as the particles thicken to large radii, causing the volume fraction to tend to the limiting value $\bar{\varphi}=\Delta_{0} / \Gamma$.

The last element of the theory is the application of the continuity equation to the particle radius distribution function $f(R, t)$

$\frac{\partial f}{\partial t}+\frac{\partial}{\partial R}\left(f \frac{\partial R}{\partial t}\right)=0$.

As shown in Appendix A, Eqs. (11), (14) and (15) can now be solved using the methods of LS or MR $[1,3,4]$ assuming a small precipitate volume fraction, leading to the next expressions for average particle size $\bar{R}(t)$, supersaturation $\Delta(t)$ and number of precipitate particles $N(t)$

$\bar{R}^{3}(t)=(2 / 3)^{2} K t+\mathrm{O}(1), \quad K=\Omega / \boldsymbol{\Phi}$ 


$$
\begin{aligned}
& \Delta(t)=\left(3 \gamma V_{\mathrm{m}}^{\beta}\right)^{2 / 3} \boldsymbol{\Phi}^{1 / 3} t^{-1 / 3}+\mathrm{O}\left(t^{-2 / 3}\right) \\
& N(t)=(3 \bar{\varphi} \boldsymbol{\Phi} / 4 \pi \gamma) t^{-1}+\mathrm{O}\left(t^{-4 / 3}\right) .
\end{aligned}
$$

To assess the effects of adding $\left(c^{\beta \alpha}\right]\left[\mu^{\beta}\right]\left[\Delta c^{\beta}\right)$ in the Gibbs-Thomson equation and in coarsening kinetics, it is frst recognised that the product of the vectors $\left(c^{\beta \alpha}\right]$ and $\left[\mu^{\beta}\right]\left[\Delta c^{\beta}\right)$ represents a dot product which accounts for the change on the Gibbs energy of the $\beta$ phase projected in the composition $\left(c^{\beta \alpha}\right]$; similarly, $\left(c^{\beta \alpha}\right]\left[\mu^{\alpha}\right]\left[\Delta c^{\alpha}\right)$ represents the Gibbs energy change of the $\alpha$ phase projected in the composition $\left(c^{\beta \alpha}\right]$. The generalised form of the Gibbs-Thomson equation is shown schematically in Fig. 2, showing that the satisfaction of Eq. (4) requires a negative value of $\left(c^{\beta \alpha}\right]\left[\mu^{\beta}\right]\left[\Delta c^{\beta}\right)$; this represents the energy "stored" in the $\beta$ phase when a curvature is present, and "released" upon precipitation as thickening occurs.

Eqs. (9) and (16) show that for $\left(c^{\beta \alpha}\right]\left[\mu^{\beta}\right]$ $\left[\Delta c^{\beta}\right)>0, R_{\mathrm{c}}$ and $K$ are larger, and the opposite is true for $\left(c^{\beta \alpha}\right]\left[\mu^{\beta}\right]\left[\Delta c^{\beta}\right)<0$; however, the maximum particle radius scaled to the critical radius remains 1.5, as in LS theory (see Appendix A). Furthermore, Eqs. (11) and (16) show that coarsening in a multicomponent system is not independent of the solution thermodynamics followed by the continuous phase due to the term $\left[\Delta c^{\beta}\right)$ represents a curve in an $n$ dimensional space which connects the initial and final states of the $\beta$ composition as coarsening progresses. This is consistent with Gibbs phase rule, which requires that for a 2 phase system where the pressure and temperature are set, the number of degrees of freedom is $n-2$. Previous investigations have reached opposite conclu-

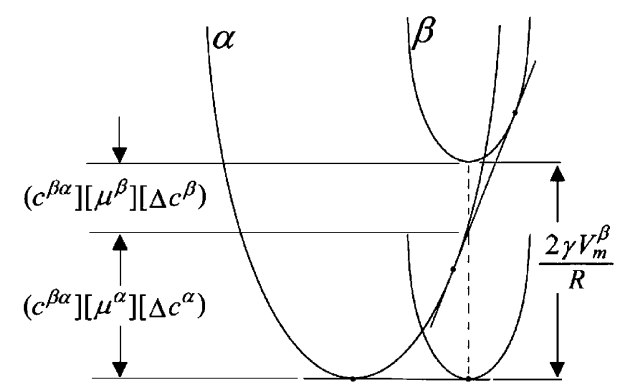

Fig. 2. Schematic representation of the equilibrium conditions required by Eq. (4). sions when the thermodynamics of $\beta$ phase are neglected [5,13].

\section{Acknowledgements}

The author is grateful to the Mexican National Council of Science and Technology (CONACYT) for financial support, and Prof. D. Fray for providing laboratory facilities.

\section{Appendix A}

Eqs. (11), (14) and (15) can be expressed using dimensionless reduced magnitudes

$\frac{\mathrm{d} \rho^{3}}{\mathrm{~d} t}=3\left(\frac{\rho}{x}-1\right)$

$\frac{\partial f}{\partial t}+\frac{\partial}{\partial \rho^{3}}\left(f v_{\rho}\right)=0$

$1=(1-\varphi) \frac{1}{x}+\frac{4 \pi}{3 V_{\mathrm{m}}^{\beta}} R_{c 0}^{3} \int_{0}^{\infty} f \rho^{3} \mathrm{~d} \rho^{3}$

where $\rho=R / R_{\mathrm{c} 0}, t=t / T, R_{\mathrm{c} 0}=\Omega / \Delta_{0}$ is the initial critical radius, $T=R_{\mathrm{c} 0}^{3} \mathbf{\Phi} / \Omega, x(t)=R_{\mathrm{c}} / R_{\mathrm{c} 0}$ is a dimensionless critical radius such that $x(0)=1$, $f\left(\rho^{3}, t\right)$ is a function which within a factor $4 \pi / 3$ is the volume distribution of the precipitates, and $v_{\rho}=\mathrm{d} \rho^{3} / \mathrm{d} t$ is the rate of growth of the precipitates in dimension space. Eqs. (A.1)-(A.3) are now expressed in terms of a reduced volume $z=\rho^{3} / x^{3}(t)$, the time expressed as $\tau=\ln x^{3}(t)$ and a new volume distribution function $\phi(z, \tau) \mathrm{d} z=f\left(\rho^{3}, t\right) \mathrm{d} \rho^{3}$ $\frac{\mathrm{d} z}{\mathrm{~d} \tau}=v(z, \gamma)=\left(z^{1 / 3}-1\right) \gamma(\tau)-z, \quad \gamma(\tau)=3 \frac{\mathrm{d} t}{\mathrm{~d} x^{3}}$

$\frac{\mathrm{d} \phi}{\mathrm{d} \tau}+\frac{\mathrm{d}}{\mathrm{d} z} \phi v(z, \gamma)=0$

$1=(1-\varphi) \mathrm{e}^{-\tau / 3}+\frac{4 \pi}{3 V_{\mathrm{m}}^{\beta}} R_{\mathrm{c} 0}^{3} \mathrm{e}^{\tau} \int_{0}^{\infty} \phi(z, \gamma) z \mathrm{~d} z$.

An asymptotic analysis of Eqs. (A.4)-(A.6) [1] shows that mass balance requires $\gamma(\tau)$ to tend to an asymptotic value $\gamma_{0}$, and the solution of Eq. (A.5) is

$\phi\left(z, \gamma_{0}\right)= \begin{cases}A \mathrm{e}^{-\tau} \mathrm{e}^{-\psi} / g\left(z, \gamma_{0}\right)=N(\tau) p\left(z, \gamma_{0}\right) & z \leqslant z_{0}=27 / 8 \\ 0 & z \geqslant z_{0}\end{cases}$ 
where $g\left(z, \gamma_{0}\right)=-v\left(z, \gamma_{0}\right) \geqslant 0, \psi=\frac{4}{3} \ln \left(z^{1 / 3}+3\right)+$ $\frac{5}{3} \ln \left(\frac{3}{2}-z^{1 / 3}\right)+\left(1-\frac{2}{3} z^{1 / 3}\right)^{-1}-\ln \left(3^{3} 2^{-5 / 2} \mathrm{e}\right), \quad A \simeq$ $0.22 \Delta_{0} / R_{c 0}^{3}$

$N(\tau)=\int_{0}^{\infty} \phi(z, \tau) \mathrm{d} z=A \mathrm{e}^{-\tau}$

is the number of grains per unit volume, and

$p\left(z, \gamma_{0}\right)=\left\{\begin{array}{cc}\mathrm{e}^{-\psi} / g\left(z, \gamma_{0}\right)=3^{3} 2^{-5 / 3} \mathrm{e}\left(z^{1 / 3}+3\right)^{-7 / 3} & \\ \left(\frac{3}{2}-z^{1 / 3}\right)^{-11 / 3} \exp \left(-\left(1-\frac{2}{3} z^{1 / 3}\right)^{-1}\right) & z \leqslant z_{0} \\ 0 & z \geqslant z_{0}\end{array}\right.$

is the probability that a grain shall have a reduced volume between $z$ and $z+\mathrm{d} z$. LS [1] showed that $p\left(z, \gamma_{0}\right)$ vanishes for values of $z^{1 / 3}$ approximately larger than 1.5, this result remains unaltered in the present analysis.

The total number of particles and average radius are obtained by inserting the original parameters in Eqs. (A.4)-(A.8).

\section{References}

[1] Lifshitz IM, Slyozov VV. J Phys Chem Solids 1961;19: $35-50$.

[2] Wagner C. Z Electrochem 1961;65:581-91.

[3] Umanstev A, Olson GB. Scripta Metal Mater 1993; 29 : 1135-40.

[4] Marqusee JA, Ross J. J Chem Phys 1983;79:373-8.

[5] Morral JE, Purdy GR. Scripta Metal Mater 1994;30:905-8.

[6] Trivedi RK. In: Aaronson HI, editor. Lectures on the theory of phase transformations. Warrendale, Pa: AIME; 1975. p. 58.

[7] Kulkarni N, DeHoff RT. Acta Mater 1997;45:4963-8.

[8] DeHoff RT. Thermodynamics in materials science. London: NewYork, Mc-Graw Hill; 1993.

[9] Lee HM, Allen SM, Grujicic M. Metal Trans A 1991; 22:2863-8.

[10] Lee HM, Allen SM, Grujicic M. Metal Trans A 1991; 22:2869-76.

[11] Lee HM, Allen SM. Metal Trans A 1991;22:2877-88.

[12] Lupis CHP. Chemical thermodynamic of materials. NY: Elsevier/North-Holland; 1983.

[13] Purdy GR. Metal Sci J 1974;8:274-6. 